\title{
Peristaltic Pumping of Solid Particles Immersed in a Viscoelastic Fluid
}

\author{
J. Chrispell ${ }^{1,2 *}$ and L. Fauci ${ }^{1,2}$ \\ ${ }^{1}$ Department of Mathematics, Tulane University, New Orleans, Louisiana 70118, USA \\ ${ }^{2}$ Center for Computational Science, Tulane University, New Orleans, Louisiana 70118, USA
}

\begin{abstract}
Peristaltic pumping of fluid is a fundamental method of transport in many biological processes. In some instances, particles of appreciable size are transported along with the fluid, such as ovum transport in the oviduct or kidney stones in the ureter. In some of these biological settings, the fluid may be viscoelastic. In such a case, a nonlinear constitutive equation to describe the evolution of the viscoelastic contribution to the stress tensor must be included in the governing equations. Here we use an immersed boundary framework to study peristaltic transport of a macroscopic solid particle in a viscoelastic fluid governed by a Navier-Stokes/Oldroyd-B model. Numerical simulations of peristaltic pumping as a function of Weissenberg number are presented. We examine the spatial and temporal evolution of the polymer stress field, and also find that the viscoelasticity of the fluid does hamper the overall transport of the particle in the direction of the wave.
\end{abstract}

Key words: viscoelastic fluid, peristaltic pumping, Oldroyd-B

AMS subject classification: 76Z05, 92C35

\section{Introduction}

Peristaltic pumping, the transport of a fluid in a tube due to waves of contraction, is fundamental to many physiological flows. Peristaltic contractions in the oviduct and uterus contribute to ovum transport and embryo implantation in the uterus $[4,11,10,13,23]$, and peristaltic contractions are responsible for the passage of urine from the kidneys to the bladder [5]. In some instances,

${ }^{*}$ Corresponding author. E-mail: jchrispe@tulane.edu 
particles of appreciable size compared to the tube diameter are transported along with the fluid. For example, the embryo diameter at implantation is about 150 microns, whereas the uterine channel diameter is on the order of 1000 microns [10]. Similarly, peristalsis of urine through the ureter can sometimes be accompanied by particles such as kidney stones [21].

In this manuscript, we present a model of the transport of a single, macroscopic, solid particle in a 2D peristaltic channel. Since many biological fluids have suspended microstructures, they may exhibit complicated, non-Newtonian responses. For this reason, we consider a simple model of a viscoelastic fluid within the channel. We describe a mathematical model and numerical method that couples the motion of moving boundaries to a Navier-Stokes/Oldroyd-B description of an elastic Boger fluid using an immersed boundary framework [27]. In particular, we extend the immersed boundary model of peristaltic pumping of a Stokes viscoelastic fluid [31] to include inertial effects, and the immersed boundary model of pumping of a solid particle within a Newtonian fluid [12] to include viscoelastic effects.

Many fluid dynamical studies of peristalsis, both analytical and numerical, have been examined in the last decades, e.g. [10, 25, 20, 19, 28, 30]. A recent analytical study of particle motion in a peristaltic fluid flow was presented in [21]. A long wavelength perturbation method is used for the fluid, along with the Basset-Boussinesq-Oseen equation for the small spherical particles. Particular application to the transport of calcium renal stones from the kidney to the ureter is studied. In this analysis, the fluid motion did influence the motion of the particles, but the particles did not affect the motion of the fluid. An experimental study of macroscopic particle transport in a peristaltic channel was presented in [18]. Immersed boundary simulations of the transport of a macroscopic particle in a Newtonian fluid were performed in [12], and a lattice Boltzmann model of this same system was recently presented [8].

Here, we study the transport of a single solid particle in a peristaltic channel with two-way coupling between the viscoelastic fluid and the particle. We find that the viscoelasticity of the fluid does hamper the overall transport of the particle in the direction of the wave. We examine the trajectories of the particle at different Weissenberg numbers and the temporal and spatial evolution of the viscoelastic stress field.

\section{Mathematical Model}

We choose an Oldroyd-B model that is derived from a microscopic description of viscoelasticity where the fluid is treated as a dilute suspension of polymers in a Newtonian solvent [3, 24]. The transport and distension of this immersed polymer field generates extra stress on the Newtonian solvent. The Navier-Stokes/Oldroyd-B equations that model the conservation of mass and momentum of this incompressible, viscoelastic fluid are: 


$$
\begin{aligned}
\boldsymbol{\sigma}+r_{t}\left(\frac{\partial \boldsymbol{\sigma}}{\partial t}+\boldsymbol{u} \cdot \nabla \boldsymbol{\sigma}-(\nabla \boldsymbol{u}) \boldsymbol{\sigma}-\boldsymbol{\sigma}(\nabla \boldsymbol{u})^{T}\right)-2 \mu_{p} \mathbf{d}(\boldsymbol{u}) & =\mathbf{0} \text { in } \Omega \\
\rho\left(\frac{\partial \boldsymbol{u}}{\partial t}+\boldsymbol{u} \cdot \nabla \boldsymbol{u}\right)+\nabla p-\mu_{s} \Delta \boldsymbol{u}-\nabla \cdot \boldsymbol{\sigma} & =\mathbf{f} \text { in } \Omega \\
\nabla \cdot \boldsymbol{u} & =0 \text { in } \Omega
\end{aligned}
$$

Here $\boldsymbol{u}$ is the fluid velocity, $p$ is pressure, $\boldsymbol{\sigma}$ is the viscoelastic contribution to the stress tensor, $\mathbf{d}(\boldsymbol{u})=\frac{1}{2}\left(\nabla \boldsymbol{u}+\nabla \boldsymbol{u}^{T}\right)$ is the fluid deformation tensor, $r_{t}$ is the polymer relaxation time, $\mu_{s}$ is the solvent viscosity, and $\mu_{p}$ denotes the polymer contribution to the zero-shear-rate viscosity.

Denoting dimensionless quantities with a prime and $\mu_{0}=\mu_{s}+\mu_{p}$, a dimensionless NavierStokes/Oldroyd-B model is obtained by the change of variables:

$$
\boldsymbol{\sigma}^{\prime}=\frac{L \boldsymbol{\sigma}}{\mu_{0} U}, \quad x^{\prime}=\frac{x}{L}, \quad \boldsymbol{u}^{\prime}=\frac{\boldsymbol{u}}{U}, \quad t^{\prime}=\frac{t U}{L}, \quad p^{\prime}=\frac{L p}{\mu_{0} U}, \quad \mathbf{f}^{\prime}=\frac{\mathbf{f} L^{2}}{\mu_{0} U},
$$

where $L$ and $U$ are characteristic length and velocity scales respectively. The non-dimensional Navier-Stokes/Oldroyd-B system is then (dropping primes):

$$
\begin{aligned}
\boldsymbol{\sigma}+\lambda\left(\frac{\partial \boldsymbol{\sigma}}{\partial t}+\boldsymbol{u} \cdot \nabla \boldsymbol{\sigma}-(\nabla \boldsymbol{u}) \boldsymbol{\sigma}-\boldsymbol{\sigma}(\nabla \boldsymbol{u})^{T}\right)-2 \beta \mathbf{d}(\boldsymbol{u}) & =\mathbf{0} \text { in } \Omega \\
R e\left(\frac{\partial \boldsymbol{u}}{\partial t}+\boldsymbol{u} \cdot \nabla \boldsymbol{u}\right)+\nabla p-(1-\beta) \Delta \boldsymbol{u}-\nabla \cdot \boldsymbol{\sigma} & =\mathbf{f} \text { in } \Omega \\
\nabla \cdot \boldsymbol{u} & =0 \text { in } \Omega
\end{aligned}
$$

where

$$
R e=\frac{L U \rho}{\mu_{0}}, \quad \lambda=\frac{r_{t} U}{L}, \quad \beta=\frac{\mu_{p}}{\mu_{0}} .
$$

Here $\lambda$ is the Weissenberg number, Re is the Reynolds number, and $\beta \in(0,1)$ denotes the fraction of the total viscosity that is viscoelastic.

In order to model the coupling of the viscoelastic fluid with the motion of the channel walls and the solid particle, we represent each structure as a Lagrangian immersed boundary $\Gamma$ whose time dependent configuration is given by $\boldsymbol{X}(\xi, t)$. Each immersed boundary transmits force to the surrounding fluid due to its elastic properties:

$$
\mathbf{f}(\boldsymbol{x}, t)=\int_{\Gamma} \boldsymbol{F}(\boldsymbol{X}, t) \delta(\boldsymbol{x}-\boldsymbol{X}(\xi, t)) d \xi
$$

Here $\delta$ is the 2D Dirac delta function. We will describe the choice of $\boldsymbol{F}$ in detail in Section 3. We also require that the immersed boundaries move with the fluid, and enforce the no-slip condition:

$$
\boldsymbol{u}(\boldsymbol{X}(\xi, t), t)=\frac{\partial \boldsymbol{X}(\xi, t)}{\partial t}=\int_{\Omega} \boldsymbol{u}(\boldsymbol{x}, t) \delta(\boldsymbol{x}-\boldsymbol{X}(\xi, t)) d \boldsymbol{x} .
$$

Equations (2.7) and (2.8) couple the Lagrangian descriptions of the immersed boundaries with the Eulerian description of the fluid and viscoelastic stress. 


\section{Numerical Methods}

Following [12, 31], we model peristaltic pumping in a channel using an immersed boundary formulation. Our computational domain is chosen to be a square whose side length is one wavelength of the channel. The channel walls extend from one end of the domain to the other, and periodic boundary conditions are used to model the infinite extent of the channel. Fluid velocity, pressure and the polymer stress tensor will be discretized on an Eulerian background grid, but the channel walls and the surface of the solid particle will be described by discrete collections of Lagrangian points.

\section{Channel walls and solid particle.}

Elastic forces chosen to enforce preferred kinematics of the channel walls and the circular geometry of the solid particle will be communicated to the background viscoelastic fluid using the Lagrangian force density $\boldsymbol{F}=\boldsymbol{F}_{w}+\boldsymbol{F}_{s}$ in (2.7). Here $\boldsymbol{F}_{w}$ is used to drive the motion of the channel walls, and $\boldsymbol{F}_{s}$ is a tensile force chosen to maintain the circular shape of the solid particle. As in [31], the force imposed by the immersed boundary walls $\boldsymbol{X}_{w}$ is:

$$
\boldsymbol{F}_{w}=-k_{w}\left(X_{w}(\eta, t)-Z(\eta, t)\right) .
$$

One may interpret these 'tether' forces as being due to very stiff springs of rest length zero connecting the wall points $\boldsymbol{X}_{w}(\eta, t)$ to target or tether points $\mathbf{Z}(\eta, t)$. The stiffness constant $k_{w}$ determines how closely the preferred shape is followed. In order to propagate a sinusoidal peristaltic wave, we choose the tether points:

$$
\mathbf{Z}(\eta, t)=\left\{\left(\eta, \frac{1}{2}+d(\eta, t)\right) \mid \eta \in[0,1]\right\} \cup\left\{\left(\eta, \frac{1}{2}-d(\eta, t)\right) \mid \eta \in[0,1]\right\}
$$

where

$$
d(\eta, t)=\frac{\alpha}{2 \pi}(1+\chi \sin (2 \pi(\eta-t))) .
$$

In (3.3), $\chi$ is the channel occlusion ratio with $\chi=0$ yielding a straight channel and $\chi=1$ yielding a completely occluded channel. The aspect ratio of the channel, $\alpha$, is taken to be 1.5 in all computations. In the simulations, we discretize the channel walls using $m_{w}$ total points equally spaced along each wall.

In order to model a solid particle whose preferred shape is a circle of radius $r$ we discretize the circle using $m_{s}$ equally spaced points $\boldsymbol{X}_{s}$. Hookean springs with stiffness $k_{s}$ and rest length determined by the initial configuration are used between each point and every other on the discretized circle to maintain a fixed solid shape. The Lagrangian force $\boldsymbol{F}_{s}$ for point $i$ on the circle is

$$
\boldsymbol{F}_{s i}=-\frac{k_{s}}{2} \sum_{j \neq i}\left(\left\|\boldsymbol{X}_{s i}-\boldsymbol{X}_{s j}\right\|-\Delta r_{i j}\right) \frac{\left(\boldsymbol{X}_{s i}-\boldsymbol{X}_{s j}\right)}{\left\|\boldsymbol{X}_{s i}-\boldsymbol{X}_{s j}\right\|},
$$

where $\Delta r_{i j}$ denotes the rest length of the spring connecting point $i$ to point $j$ on the discretized solid. How closely this elastic object maintains its circular shape may be controlled by the choice 


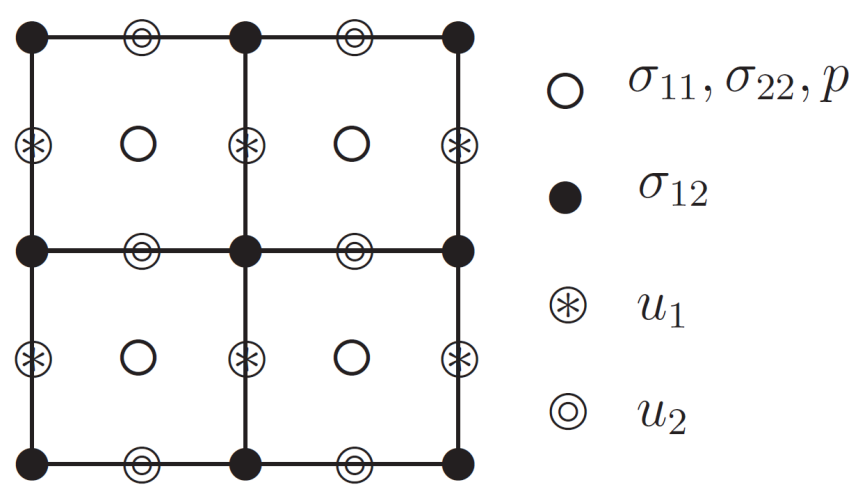

Figure 1: A MAC grid with pressure and diagonal stress components $\sigma_{11}$ and $\sigma_{22}$ stored at cell centers, the off diagonal component of the viscoelastic stress $\sigma_{12}$ is stored at cell corners, and the fluid velocity components $u_{1}$ and $u_{2}$ are stored on vertical and horizontal cell edges respectively.

of stiffness constant $k_{s}$. Note that if $k_{s}=0$, the discretized points do not exert force on the surrounding fluid, and act as passive marker particles.

\section{Eulerian discretization}

The 2D periodic domain $\Omega$ is discretized using a uniformly spaced MAC (marker and cell) grid. MAC grids store pressure and diagonal stress components at cell centers, the off diagonal component of the viscoelastic contribution to the stress $\boldsymbol{\sigma}$ is stored at cell corners, and the $x$ and $y$ components of the fluid velocity are stored at the center of vertical and horizontal cell edges respectively $[16,31]$ (See Figure 1). A nice description of the use of staggered meshes for viscoelastic fluids is given by Teran et al. in [31]. Second order finite difference techniques are used to discretize the spatial derivatives.

Finite difference evolution of viscoelastic stress often exhibits grid-scale oscillations in some components [15], and excessive growth in stress gradients [33]. To control polymer stress gradient growth a stabilization technique must be implemented. Here we follow [33], and add stress diffusion to the constitutive equation (2.4) with coefficient $\nu$ :

$$
\boldsymbol{\sigma}+\lambda\left(\frac{\partial \boldsymbol{\sigma}}{\partial t}+\boldsymbol{u} \cdot \nabla \boldsymbol{\sigma}-(\nabla \boldsymbol{u}) \boldsymbol{\sigma}-\boldsymbol{\sigma}(\nabla \boldsymbol{u})^{T}-\nu \Delta \boldsymbol{\sigma}\right)-2 \beta \mathbf{d}(\boldsymbol{u})=\mathbf{0}
$$

In this modified constitutive equation, we set the stress diffusion coefficient $\nu$ to be on the order of the mesh spacing squared. Adding stress diffusion in this manner does not alter the consistency of the numerical discretization. For other works discussing stress diffusion in viscoelastic flows see $[9,17,26,29]$. Other stabilization techniques are commonly used in finite element implementations of viscoelastic flow including Streamline-upwind Petrov/Galerkin (SUPG) [6, 7], and Discontinuous Galerkin (DG) solution methods [1,2]. 


\section{Temporal discretization}

At the beginning of the $n^{\text {th }}$ time step, we are given $\boldsymbol{u}^{n}, \boldsymbol{\sigma}^{n}$. We advance to $\boldsymbol{u}^{n+1}, \boldsymbol{\sigma}^{n+1}$ by using the projection method for the incompressible Navier-Stokes equations as in Kim and Moin [22] and the viscoelastic evolution algorithm as in [31].

Step 1 (Velocity solve): Solve for the intermediate velocity field $\boldsymbol{u}^{*}$. A Crank-Nicholson method and a second order Adams-Bashforth method are used to temporally discretize the viscous and convective terms:

$$
\frac{\boldsymbol{u}^{*}-\boldsymbol{u}^{n}}{\Delta t}=-\frac{1}{2}\left(3 \boldsymbol{u}^{n} \cdot \nabla \boldsymbol{u}^{n}-\boldsymbol{u}^{n-1} \cdot \nabla \boldsymbol{u}^{n-1}\right)+\frac{(1-\beta)}{2 R e} \Delta\left(\boldsymbol{u}^{*}+\boldsymbol{u}^{n}\right)-\frac{1}{R e} \nabla \cdot \boldsymbol{\sigma}^{n}+\frac{1}{R e} \mathbf{f}^{\mathbf{n}}
$$

Step 2 (Poisson step): Incompressibility is enforced by solving the Poisson problem:

$$
\Delta \hat{p}=\frac{1}{\Delta t} \nabla \cdot \boldsymbol{u}^{*}
$$

setting $\hat{p}$ at a point to insure the system is not singular. Then $\boldsymbol{u}^{n+1}=\boldsymbol{u}^{*}-\Delta t \nabla \hat{p}$.

Step 3 (Stress evolution): Writing (3.5) as

$$
\frac{\partial \boldsymbol{\sigma}}{\partial t}=\mathbf{g}(\boldsymbol{\sigma}, \boldsymbol{u})
$$

where

$$
\mathbf{g}(\boldsymbol{\sigma}, \boldsymbol{u})=\frac{2 \beta}{\lambda} \mathbf{d}(\boldsymbol{u})-\frac{1}{\lambda} \boldsymbol{\sigma}-\left(\boldsymbol{u} \cdot \nabla \boldsymbol{\sigma}-(\nabla \boldsymbol{u}) \boldsymbol{\sigma}-\boldsymbol{\sigma}(\nabla \boldsymbol{u})^{T}-\nu \Delta \boldsymbol{\sigma}\right),
$$

we explicitly update $\sigma$ using a second-order Runge-Kutta method by defining two intermediate values of the viscoelastic extra stress $\boldsymbol{\sigma}^{n+A}$ and $\boldsymbol{\sigma}^{n+B}$ :

$$
\begin{aligned}
& \boldsymbol{\sigma}^{n+A}=\boldsymbol{\sigma}^{n}+\Delta t \mathbf{g}\left(\boldsymbol{\sigma}^{n}, \boldsymbol{u}^{n+1}\right) \\
& \boldsymbol{\sigma}^{n+B}=\boldsymbol{\sigma}^{n+A}+\Delta t \mathbf{g}\left(\boldsymbol{\sigma}^{n+A}, \boldsymbol{u}^{n+1}\right)
\end{aligned}
$$

and

$$
\boldsymbol{\sigma}^{n+1}=\frac{\boldsymbol{\sigma}^{n}+\boldsymbol{\sigma}^{n+B}}{2}
$$

\section{Immersed boundary and fluid interaction}

The Eulerian force density is a Dirac delta function layer of force supported by the immersed boundaries (2.7). A regularized delta function with compact support is used to spread the Lagrangian elastic forces from the immersed boundaries to the grid. Following [27], we choose $\delta_{h}(\mathbf{x})=\phi_{h}(x) \phi_{h}(y)$ where $h$ denotes the grid spacing, and

$$
\phi_{h}(r)= \begin{cases}\frac{1}{8 h}\left(3-\frac{2|r|}{h}+\sqrt{1+\frac{4|r|}{h}-\frac{4 r^{2}}{h^{2}}}\right) & \text { if }|r| \leq h, \\ \frac{1}{8 h}\left(5-\frac{2|r|}{h}-\sqrt{-7+\frac{12|r|}{h}-\frac{4 r^{2}}{h^{2}}}\right) & \text { if } h<|r| \leq 2 h \\ 0 & \text { otherwise } .\end{cases}
$$


Denoting the discrete Lagrangian point $\boldsymbol{X}_{k}$ and its Lagrangian force density as $\boldsymbol{F}_{k}$, Equations (2.7) and (2.8) may be discretized as:

$$
\mathbf{f}_{i, j}=\sum_{k} \boldsymbol{F}_{k} \delta_{h}\left(\boldsymbol{x}_{i, j}-\boldsymbol{X}_{k}\right) \Delta l_{k}
$$

and

$$
\boldsymbol{u}_{k}=\sum_{i, j} \boldsymbol{u}_{i, j} \delta_{h}\left(\boldsymbol{x}_{i, j}-\boldsymbol{X}_{k}\right) h^{2}
$$

\section{Summary of algorithm}

Given $\boldsymbol{X}^{n}, \boldsymbol{\sigma}^{n}, \boldsymbol{u}^{n}$ at time $t^{n}=n \Delta t$ :

- Calculate the force $\boldsymbol{F}$ due to the configuration $\boldsymbol{X}^{n}$ of the immersed boundaries.

- Spread $\boldsymbol{F}$ using the regularized delta function $\delta_{h}$ to the computational grid with (3.14).

- Solve the Navier-Stokes/Oldroyd-B equations using the three steps above.

- Update the position of the immersed boundary points by interpolating the fluid-velocity at each immersed boundary point using (3.15).

The Kim-Moines projection method used for the Navier-Stokes solution is second order in both space and time, as is the Runge-Kutta advancement of the polymeric stress field. However, the use of the discretized delta functions to spread the forces of the immersed boundaries to the background grid, and to interpolate the grid velocities back to the immersed boundaries degrades the overall convergence to first order in space and time [27]. We note that variants of this implementation that regard the immersed boundary as having a finite thickness are shown to be second order [14]. We also note that since the immersed boundary forces are treated explicitly, $\Delta t$ must be chosen small enough to ensure stability of the Navier-Stokes time-stepping. In particular, as the stiffness constants used to drive the peristaltic channel and to maintain the circular particle shape are increased, the time step must be decreased.

\section{Numerical Results}

\section{Channel with no particle: mean flow calculations}

To exam the effects of viscoelasticity on the flow generated by a peristaltic pumping channel, we consider a base set of model parameters. These geometric, physical and numerical parameters are the defaults used for the simulations presented and are shown in Table 1.

The stiffness constants $k_{w}$ and $k_{s}$ are used to enforce the traveling waves along the channel walls and the circular shape of the solid particle respectively. While these values could be chosen 
to reflect material properties of the walls and particle, here we treat them as numerical parameters that are chosen high enough to give the desired kinematics of the walls and to maintain the particle shape. Because of the stiffness of the springs, the larger the values of $k_{w}$ and $k_{s}$, the smaller the time step $\Delta t$ must be for the stability of the calculations. We have found that for simulations that involve a highly-occluded channel $(\chi=0.5)$ and a significant Weissenberg number $(\lambda=5)$, it was necessary to use the smaller values of $k_{w}, k_{s}$ and $\Delta t$ presented in Table 1 . In addition, for stable evolution of the stress equation, we found that choosing $\nu=h^{2} \approx 1.525 e-5$ was satisfactory. Without this added stress diffusion, the viscoelastic calculations became unstable for long run times.

\begin{tabular}{lcc||lcc}
\hline Parameter & Symbol & value & Parameter & Symbol & value \\
\hline Domain & $\Omega$ & $(0,1) \times(0,1)$ & Solid particle radius & $r$ & 0.075 \\
Reynolds No. & $R e$ & 1 & Channel points & $m_{w}$ & 580 \\
Grid spacing & $h$ & $\frac{1}{256}$ & Particle points & $m_{s}$ & 241 \\
Time step & $\Delta t$ & $\frac{1}{1000}, \frac{1}{5000}$ & Stiffness $k_{w}$ & $k_{w}$ & 2000,4000 \\
Diffusion coefficient & $\nu$ & $h^{2}$ & Stiffness $k_{s}$ & $k_{s}$ & $1.5,2$ \\
Viscosity fraction & $\beta$ & $\frac{1}{2}$ & Aspect ratio & $\alpha$ & 1.5 \\
\hline
\end{tabular}

Table 1: Case study parameters

The primary function of a peristaltic channel is to pump fluid in the direction of the wave. The efficacy of this pumping is measured by calculating the dimensionless mean flow rate $\theta$ in the wave direction. First, we will examine the effects of the occlusion ratio $\chi$ and Weissenberg number $\lambda$ on this flow rate in the absence of an immersed solid particle. The mean flow rate is obtained by calculating the mass flux across the channel at a fixed position in the laboratory frame, $x_{f}$, over a period:

$$
\theta=\frac{\pi}{\alpha \chi\left(T_{2}-T_{1}\right)} \int_{T_{1}}^{T_{2}} \int_{\frac{1}{2}+d\left(x_{f}, t\right)}^{\frac{1}{2}-d\left(x_{f}, t\right)} u_{1}\left(x_{f}, y\right) d y d t .
$$

For a Newtonian fluid with Reynolds number zero, Jaffrin et al [20] computed the zeroth order dimensionless mean flow rate $\theta$ in the case of $\alpha \ll 1$ as

$$
\theta_{S}=\frac{3 \chi}{2+\chi^{2}}
$$

This result was later extended to second order in $\alpha$ by Jaffrin and Shapiro in [19]:

$$
\theta_{J}=\frac{15 \chi^{2}+2 \alpha^{2}\left(4\left(1-\chi^{2}\right)^{\frac{5}{2}}+\left(7 \chi^{2}-4\right)\left(1-\chi^{2}\right)\right)}{\chi\left(5\left(2+\chi^{2}\right)+6 \alpha^{2} \chi^{2}\left(1-\chi^{2}\right)\right)} .
$$

In our non dimensional channel, the period of the wave is one time unit. The mean flow rate $\theta$ over this unit period can be computed as a function of the start time for the periodic movement, $t_{0}$, 
at a fixed position in the laboratory frame $x_{f}$, and is given by:

$$
\theta\left(t_{0}\right)=\frac{\pi}{\alpha \chi} \int_{t_{0}}^{t_{0}+1} \int_{\frac{1}{2}+d\left(x_{f}, t\right)}^{\frac{1}{2}-d\left(x_{f}, t\right)} u_{1}\left(x_{f}, y\right) d y d t .
$$

Because of the evolution of the viscoelastic stress, the nonzero Weissenberg calculations take some time to reach steady state. To illustrate this transient behavior, we consider flow rates averaged over a period for two different starting times, $t_{0}=0.2$ and $t_{0}=19$. We choose a non-zero start time to allow the Newtonian case to reach a steady, periodic state. The mean flow for different occlusion ratios and for Weissenberg numbers $\lambda=0,0.5,1,2$, and 5 are shown in Figure 2 . While we report the mean flow rate computed at cross-section $x_{f}=0.5$ of the channel, due to incompressibility, the average flow rate is the same at all cross-sections. Figure 2 also shows a comparison with the asymptotic analysis of Jaffrin and Shapiro [19]. Note that the asymptotic values of mean flow predicted using a Stokes flow assumption agree very well with the calculated values computed for the Newtonian case $\lambda=0, R e=1$. We remark that the results presented here illustrate that the addition of inertial terms gives a slight increase in the flow rates, as computed for the Stokes/Oldroyd-B case in [31]. When $t_{0}=19$ (the twentieth period of motion for the walls), the viscoelastic cases have reached their steady, periodic state. The flow rate values computed for the Newtonian simulations during the twentieth period are indistinguishable from those computed during the early period. However, this is clearly not the case for the $\lambda=5$ calculation. As observed in [31], the maximum mean flow rate at this higher Weissenberg number is achieved at a value of $\chi$ well before complete occlusion. We also see a striking change in the ordering of flow rates at a fixed occlusion ratio with respect to Weissenberg number when examining the values computed with $t_{0}=0.2$ versus $t_{0}=19$. During the early period, the Newtonian case gives the largest flow rate, followed by $\lambda=5$ monotonically down to $\lambda=0.5$. During the twentieth period, this is entirely reversed, with a perfect ordering of decreasing flow rate as Weissenberg number is increased.

To examine this reversal further, we plot the value of periodic mean flow rate $\theta\left(t_{0}\right)$ as a function of starting time for two occlusion ratios $(\chi=0.1$ and $\chi=0.4)$ in Figure 3. The Newtonian $(\lambda=0)$ case is seen to obtain a steady periodic mean flow rate almost instantaneously. The mean flow rate in the channel with greater occlusion ratio takes longer to achieve steady state, as do the higher Weissenberg number flows.

\section{Channel with solid particle}

We now illustrate the dynamics of transport in a peristaltic channel with an immersed solid particle within a Navier-Stokes/Oldroyd-B fluid with $R e=1$ and $\lambda=5$. Figure 4 shows a sequence of snapshots of a particle of radius $r=0.075$ initially placed along the centerline of a channel with occlusion ration $\chi=0.4$ and aspect ratio $\alpha=1.5$. In order to interpret the directions and degree of distension of the polymer field, we examine the scaled and shifted viscoelastic stress field

$$
\hat{\boldsymbol{\sigma}}=\frac{\lambda}{\beta} \boldsymbol{\sigma}+\mathbf{I}
$$



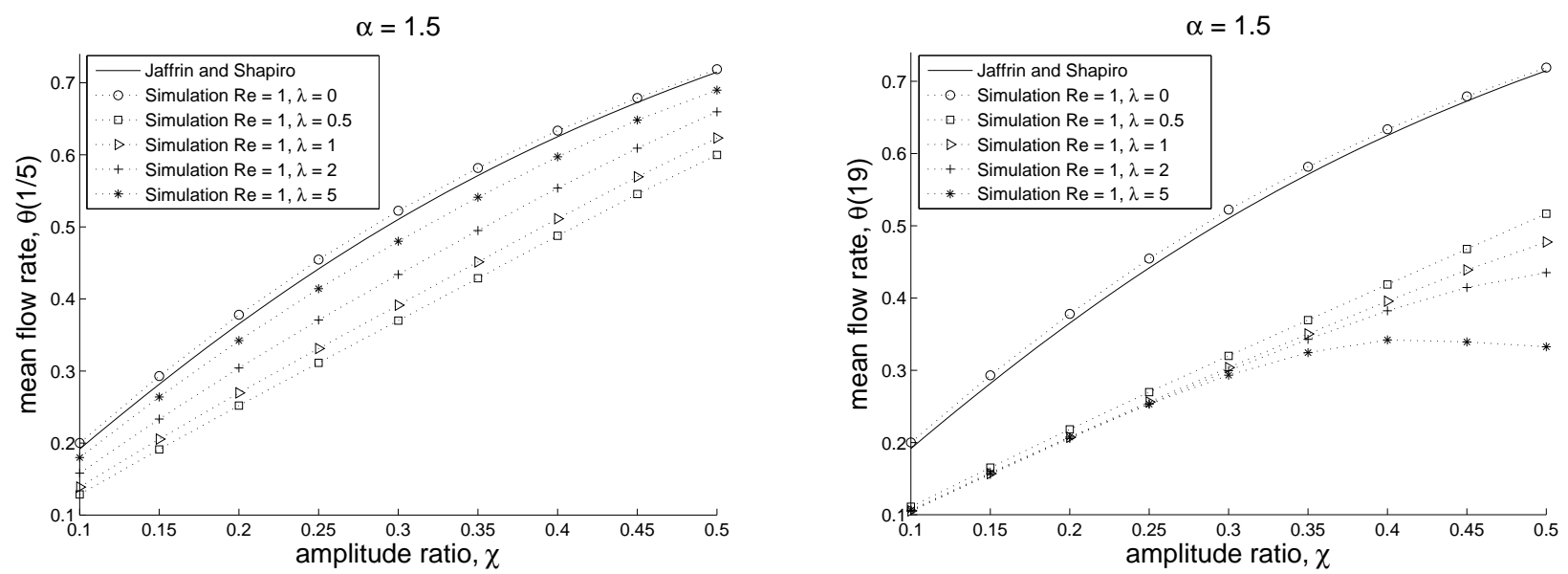

Figure 2: Mean flow rate vs. occlusion ratio for different Weissenberg numbers for period of wave starting at $t=.2$ (left) and $t=19$ (right).

$\alpha=1.5, \chi=0.1$

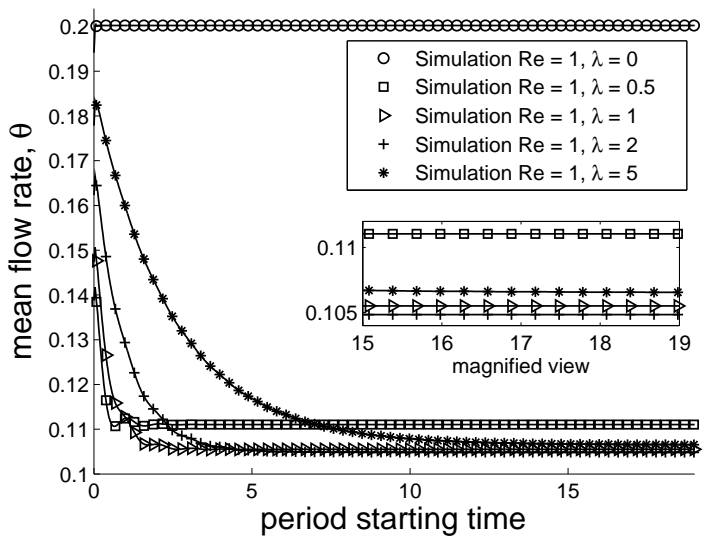

$\alpha=1.5, \chi=0.4$

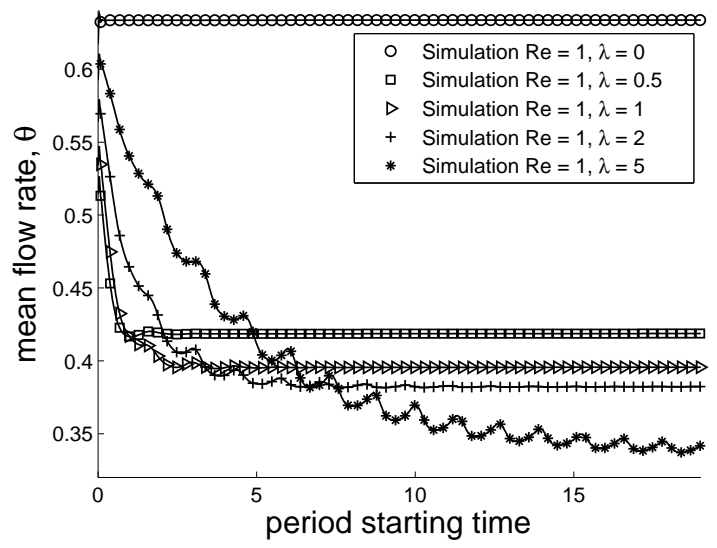

Figure 3: Mean flow rate per period vs. period start time at occlusion ratios $\chi=0.1$ (left) and $\chi=0.4$ (right). 
where I denotes the identity tensor. This shifted stress tensor arises from alternate, but equivalent, scalings of the Oldroyd-B equations and has been used in [31,32]. Here, the trace of $\hat{\sigma}$ is the meansquare distension of the microscopic polymer coils. The tensor $\hat{\sigma}$ is symmetric positive definite, and therefore has two positive eigenvalues and corresponding orthogonal unit eigenvectors. We may visualize the stress field by plotting ellipses whose orientation and aspect ratio are determined by the eigenvalues and eigenvectors of the stress tensors at grid points. These ellipses are aligned with the local polymer distension in the fluid. The shifted stress tensor $\hat{\sigma}$ represents the initial isotropic stress $\boldsymbol{\sigma}=\mathbf{0}(\hat{\boldsymbol{\sigma}}=\mathbf{I})$ as a circles of radius 1, as seen in the time zero frame of Figure 4. A consistent scaling of the ellipse axes is used when displaying all frames in Figure 4, showing the evolution of the stress field as the wave travels to the right. Looking at early times, $(t=$ $0.1,0.2,0.3)$ it is evident that in the portion of the channel where the walls are moving inwards (note velocity vectors), the stress ellipses become elongated in the x-direction. Similarly, in the portion of the channel where the walls are moving outwards, the stress ellipses are elongated in the y-direction. As the movement of the channel walls continue, the maximal polymer distension occurs in the narrowest portion of the channel.

Since we are using an immersed boundary framework to model the solid particle, the particle itself is filled with the same viscoelastic fluid in its interior as that on its exterior. Because there is little deformation of the particle, the polymer stress ellipses in its interior undergo only small changes.

Figure 4 shows that the particle is transported towards the right, in the direction of wave motion, in a non-monotonic manner. The particle is moving rightwards until the frame $t=0.6$, but then reverses direction as it approaches the neck of the channel (or as the contraction wave passes over it). It is then carried to the left until just before $t=1.0$. Because the particle's center was placed directly on the centerline of the channel, there is no lateral displacement due to symmetry. Figure 5 examines the effect of the presence of the particle on the polymer stress fields for times $t=1,4$ and 12. Contours of the trace of $\boldsymbol{\sigma}$ are depicted in a channel with no particle (top row) and in a channel with the particle (bottom row). For each fixed time, the contours are scaled consistently. It is evident that the maximal polymer distension is in the contracted region of the channel, with and without the particle. However, the presence of the particle does magnify this distension. Moreover, it is when the particle is in the narrowest region that it is transported backwards. The stress build-up in the contraction increases the velocity of this backwards motion.

We examine the effect of Weissenberg number on the particle trajectory in Figure 6, where the $\mathrm{x}$-position of the centroid of the particle is plotted as a function of time for $\lambda=0,0.5,1,2$, and 5 . For very early times, each trajectory basically overlaps, because the polymer stress is initially isotropic and uniform. However, the paths quickly diverge. The particle moving in the Newtonian fluid enjoys maximal transport. The upper inset in Figure 6 shows that for early times, while the Newtonian particle exhibits the largest displacement, it is followed by $\lambda=5$ monotonically down to $\lambda=0.5$. The lower inset shows that this is reversed for later times, when a steady state has been achieved for all Weissenberg numbers.

Note that the trajectories of the $\mathrm{x}$-component of the centroids each evolve to temporally periodic states, but with different periods. We see that the Newtonian particle spends longer time progressing forward within its period compared to the particles in the viscoelastic fluids. We examine this 

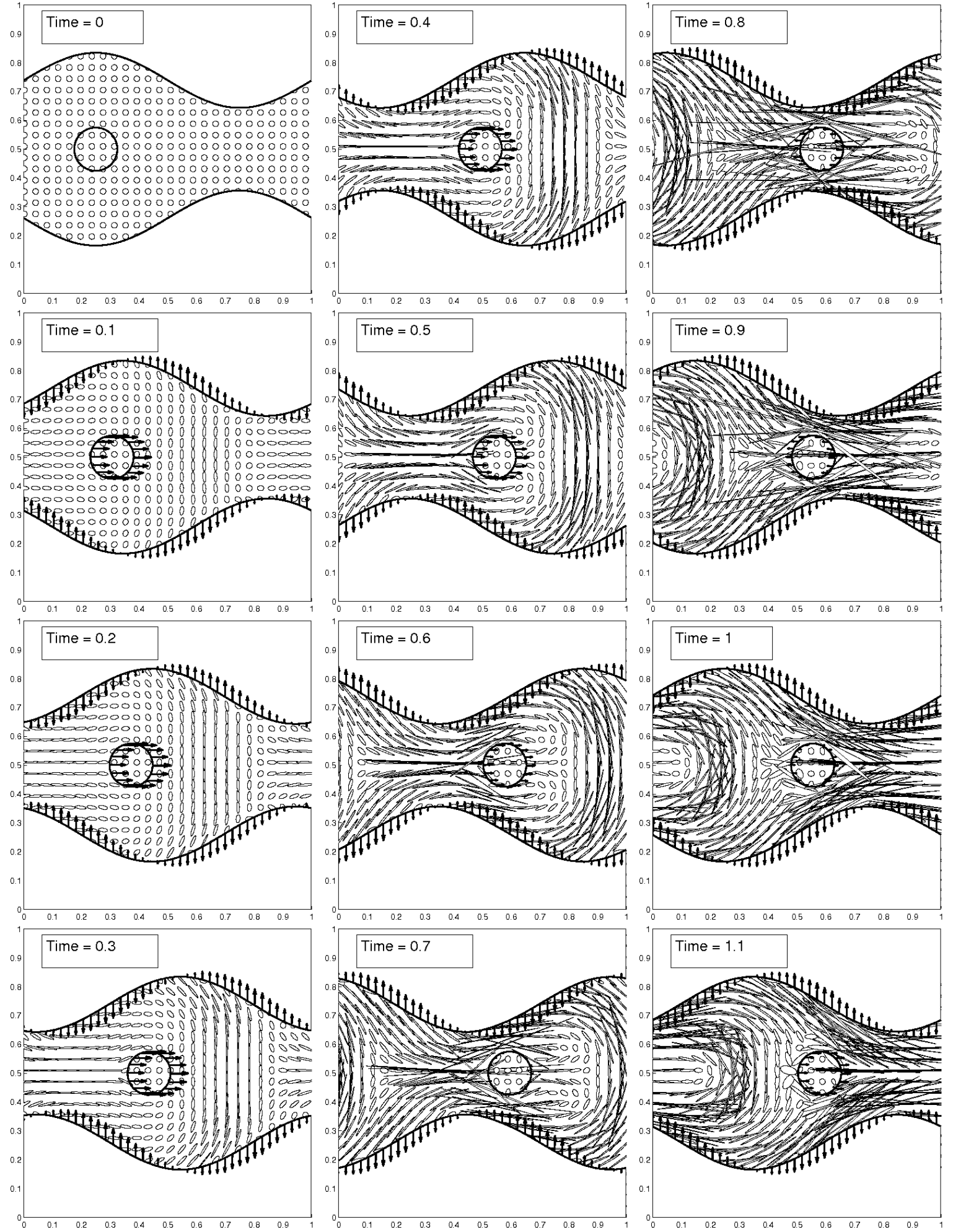

Figure 4: Evolution of stress ellipses during the first period of pumping for a viscoelastic fluid with $R e=1, \lambda=5$. Here the occlusion rato is $\chi=0.4$, the aspect ratio of the unit wavelength channel is $\alpha=1.5$ and the radius of the particle is $r=0.075$. 

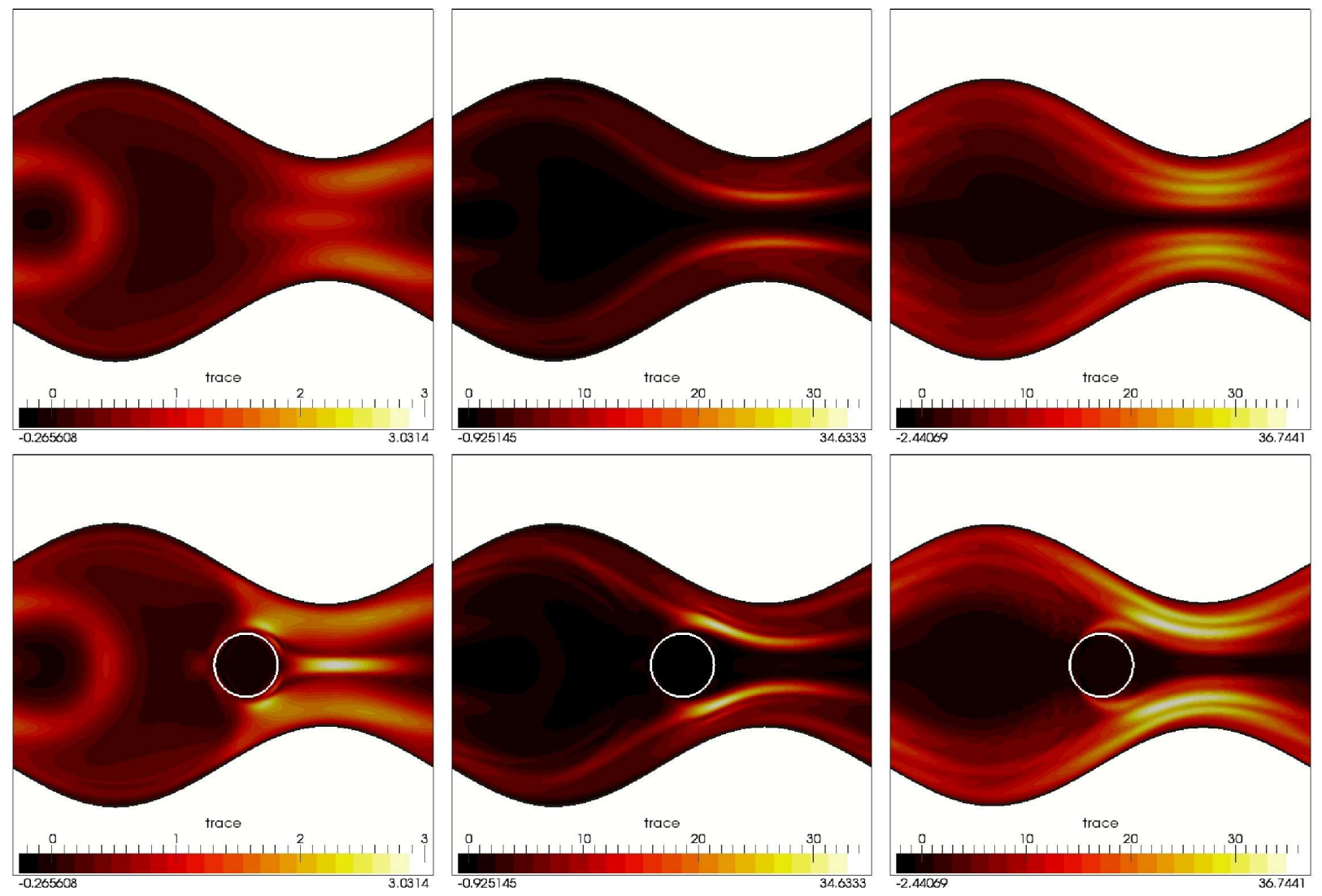

Figure 5: Columns show contours of $\operatorname{tr}(\boldsymbol{\sigma})$ at $t=1, t=4$, and $t=12$ without (top) and with a solid particle (bottom). Here the occlusion rato is $\chi=0.4$, the aspect ratio of the unit wavelength channel is $\alpha=1.5$ and the radius of the particle is $r=0.075$.

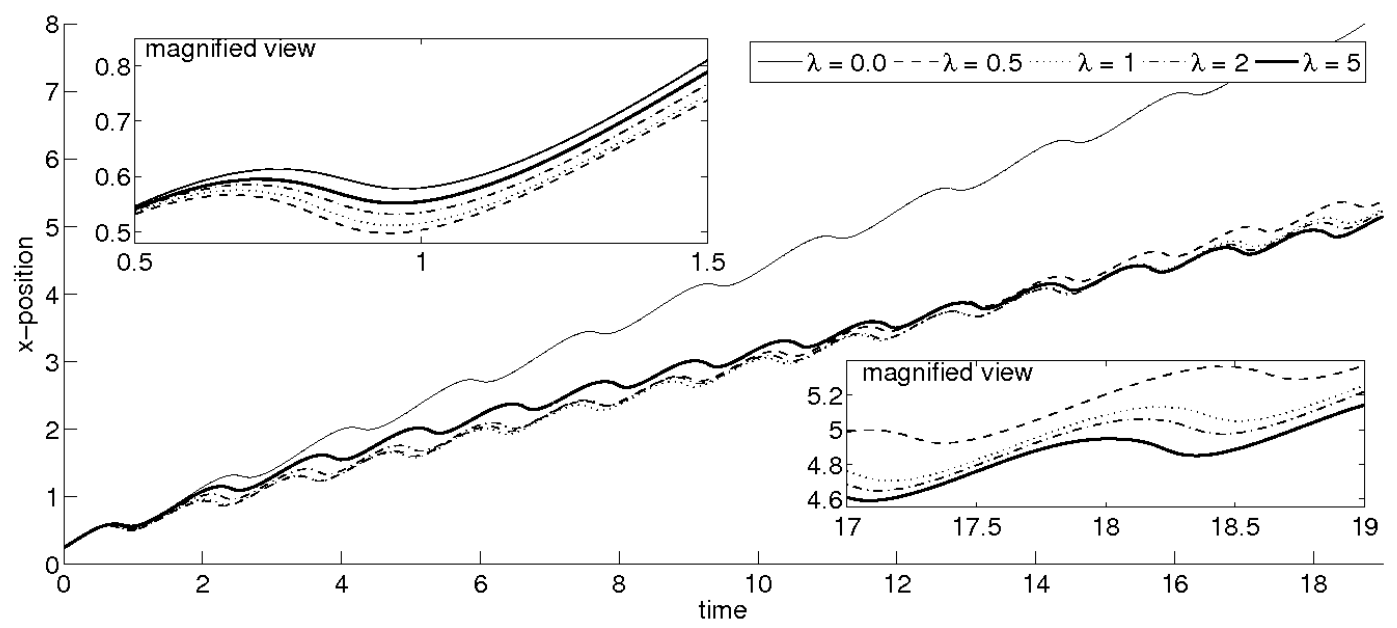

Figure 6: Trajectory of x-position of centroid of solid particle placed initially at $x=0.25$ in the channel for Weissenberg numbers $\lambda=0,0.5,1,2,5$. 
in more detail by looking at how the period of the particle evolves over time. (This information may be extracted from the trajectory information by tabulating the times at which the x-centroid of the particle changes direction from moving in a positive direction to a negative direction.) Figure 7 shows the time evolution of period length of the trajectories for $\lambda=0,0.5,1,2,5$ (on the left), as well as the time evolution of the fraction of that period where the particle has negative velocity (on the right). The period length for of the movement of the solid particle in the Newtonian fluid is immediately established, and is about 1.7 times the unit speed of the wave of contraction being passed along the channel walls. Note that the higher Weissenberg numbers take longer to reach their steady periodic state, as expected. We also see a dramatic difference in time spent going backwards, increasing from just about $12 \%$ for the Newtonian case to over $25 \%$ for the most viscoelastic fluid $\lambda=5$.
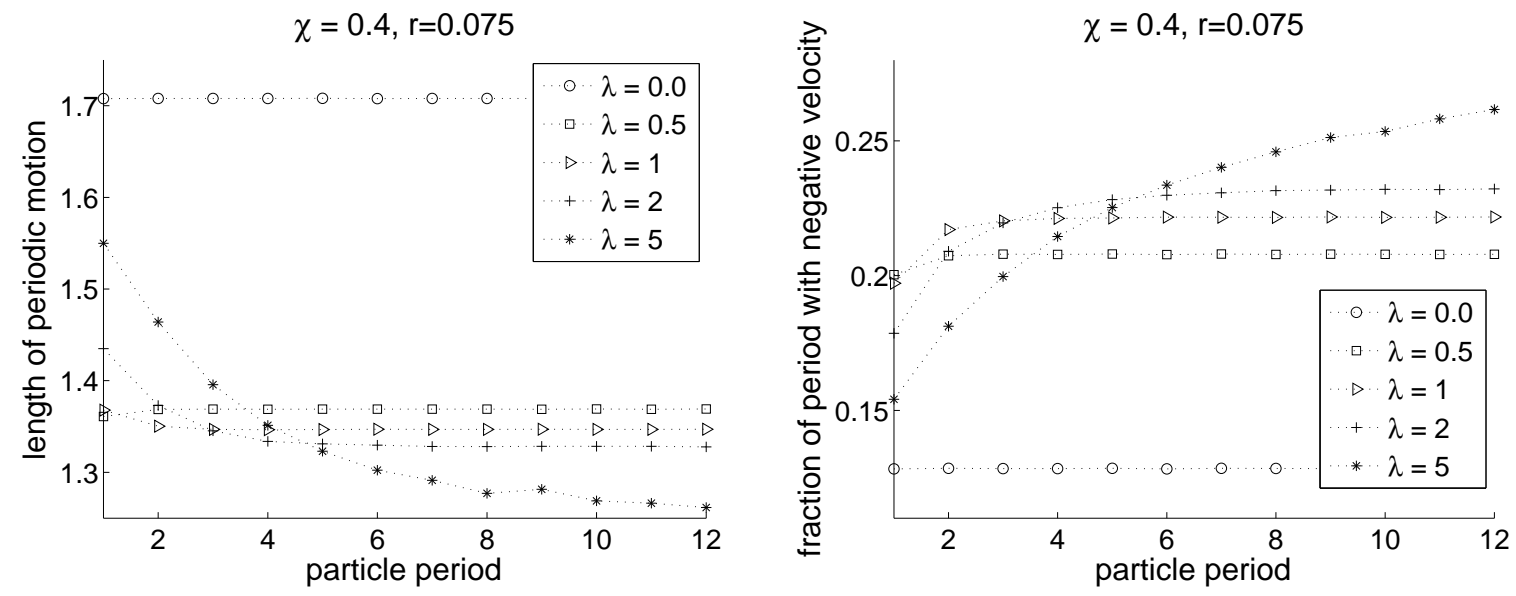

Figure 7: The temporal period of the solid particle as a function of starting time for Weissenberg numbers $\lambda=0,0.5,1,2,5$ (left). The fraction of each period that the particle experienced negative velocity in the $\mathrm{x}$-direction for Weissenberg numbers $\lambda=0,0.5,1,2,5$ (right).

\section{Conclusions}

These numerical studies of the peristaltic pumping of a macroscopic solid particle show that there is a marked difference in transport when the surrounding fluid is Newtonian compared to viscoelastic. The presence of viscoelasticity impedes the forward motion of the particle, and enhances the backward motion. We have demonstrated that complex stress patterns evolve in the peristaltic channel, and that these patterns are also influenced by the presence of the particle.

Here, we have chosen to focus on the symmetric case of a single particle placed along the centerline of the channel at the single Reynolds number $R e=1$. Future investigations will examine the effect of particle diameter, Reynolds number, and initial particle placement. Moreover, we expect that the pumping of a group of macroscopic solid particles in this viscoelastic fluid will give result to complex collective dynamics. 


\section{Acknowledgements}

This work was supported by NSF Grant No. DMS-0652775.

\section{References}

[1] J. Baranger, A. Machmoum. Existence of approximate solutions and error bounds for viscoelastic fluid flow: characteristics method. Comput. Methods Appl. Mech. Engrg. , 148 (1997), No. 1-2, 39-52.

[2] J. Baranger, D. Sandri. Finite element approximation of viscoelastic fluid flow: existence of approximate solutions and error bounds. I. Discontinuous constraints. Numer. Math., 63 (1992), No. 1, 13-27.

[3] R.B. Bird, R.C. Armstrong, O. Hassager. Dynamics of Polymeric Liquids. WileyInterscience, 1987.

[4] J.R. Blake, P.G. Vann, H Winet. A model of ovum transport. J. Theor. Biol., 102 (1983), No. $1,145-166$.

[5] S. Boyarski, C. Gottschalk, E. Tanagho, P. Zimskind. Urodynamics: Hydrodynamics of the Ureter and the Renal Pelvis. Academic Press, New York, 1971.

[6] A. Brooks, T. Hughes. Streamline Upwind/Petrov-Galerkin formulations for convection dominated flows with particular emphasis on the incompressible Navier-Stokes equations. Computer Methods in Applied Mechanics and Engineering, 32 (1982), No. (1-3), 199 - 259.

[7] J.C. Chrispell, V.J. Ervin, E.W. Jenkins. A fractional step [theta]-method approximation of time-dependent viscoelastic fluid flow. Journal of Computational and Applied Mathematics, 232 (2009), No. 2, 159 - 175.

[8] K. Connington, Q. Kang, H. Viswanathan, A. Abdel-Fattah, S. Chen. Peristaltic particle transport using the lattice boltzmann method. Phys. of Fluids, 21 (2009), No. 5, 053301.

[9] A.W. El-Kareh, L.G. Leal. Existence of solutions for all deborah numbers for a nonNewtonian model modified to include diffusion. Journal of Non-Newtonian Fluid Mechanics, 33 (1989), No. 3, 257 - 287.

[10] O. Eytan, D. Elad. Analysis of intra-uterine fluid motion induced by uterine contractions. Bull. Math. Biol., 61 (1999), No. 2, 221-238.

[11] O. Eytan, A.J. Jaffa, J. Har-Toov, E. Dalach, D. Elad. Dynamics of the intrauterine fluid-wall interface. Ann. Biomed. Engr., 27 (1999) No. 3, 372-9.

[12] L. Fauci. Peristaltic pumping of solid particles. Comp. \& Fluids, 21 (1992), No. 4, 583-598. 
[13] L. Fauci, R. Dillon. Biofluidmechanics of reproduction. Annu. Rev. Fluid. Mech., 38 (2006), No. 1, 371-394.

[14] B.E. Griffith, C.S. Peskin. On the order of accuracy of the immersed boundary method: Higher order convergence rates for sufficiently smooth problems. Journal of Computational Physics, 208 (2005), No. 1, 75 - 105.

[15] R. Guy, A. Fogelson. A wave propagation algorithm for viscoelastic fluids with spatially and temporally varying properties. Comput. Methods Appl. Mech. Engr., 197 (2008), No. 1, 2250-2264.

[16] F. H. Harlow, J. E. Welch. Numerical calculation of time-dependent viscous incompressible flow of fluid with free surface. Phys. of Fluids, 8 (1965), No. 12, 2182-2189.

[17] E. J. Hinch. Uncoiling a polymer molecule in a strong extensional flow. Journal of NonNewtonian Fluid Mechanics, 54 (1994), No. C, 209-230.

[18] T.K. Hung, T.D. Brown. Solid-particle motion in two-dimensional peristaltic flows. J. Fluid Mech, 73 (1976), No. 1,77-96.

[19] M. Y. Jaffrin and A. H. Shapiro. Peristaltic pumping. Annu. Rev. Fluid Mech., 3 (1971), No. $1,13-37$.

[20] M. Y. Jaffrin, A. H. Shapiro, S. L. Weinberg. Peristaltic pumping with long wavelengths at low reynolds number. J. Fluid Mech., 37 (1969), No. 4, 799-825.

[21] J. Jimenez-Lozano, M. Sen, P. Dunn. Particle motion in unsteady two-dimensional peristaltic flow with application to the ureter. Phys. Rev. E, 79 (2009), No. 4, 041901.

[22] J. Kim, P. Moin. Application of a fractional-step method to incompressible Navier-Stokes equations. J. Comp. Physics, 59 (1985), No. 2, 308 - 323.

[23] G. Kunz, D. Beil, H. Deiniger, A. Einspanier, G. Mall, G. Leyendecker. The uterine peristaltic pump. normal and impeded sperm transport within the female genital tract. Adv. Exp. Med. Biol., 424 (1997), No. 1, 267-277.

[24] R.G. Larson. The Structure and Rheology of Complex Fluids. Oxford University Press, 1998.

[25] M. Li, J. Brasseur. Non-steady peristaltic transport in finite-length tubes. J. Fluid Mech., 248 (1993), No. 1, 129-151.

[26] C.Y. Lu, P.D. Olmsted, R.C. Ball. Effects of nonlocal stress on the determination of shear banding flow. Phys. Rev. Lett., 84 (2000), No. 4, 642-645.

[27] C.S. Peskin. The immersed boundary method. Acta Numerica, 11 (2002), 479-517.

[28] C. Pozrikidis. A study of peristaltic flow. J. Fluid Mech. 180 (1987), 180:515. 
[29] J.M. Rallison. Dissipative stresses in dilute polymer solutions. Journal of Non-Newtonian Fluid Mechanics, 68 (1997), No. 1, 61 - 83.

[30] S. Takabatake, K. Ayukawa, A. Mori. Peristaltic pumping in circular cylindrical tubes: a numerical study of fluid transport and its efficiency. J. Fluid Mech., 194 (1988), 193:267.

[31] J. Teran, L. Fauci, M. Shelley. Peristaltic pumping and irreversibility of a Stokesian viscoelastic fluid. Phys. of Fluids, 20 (2008), No. 7, 073101.

[32] J. Teran, L. Fauci, M. Shelley. Viscoelastic fluid response can increase the speed and efficiency of a free swimmer. Phys. Rev. Letters, 104 (2010), No. 3, 038101.

[33] B. Thomases, M. Shelley. Transition to mixing and oscillations in a Stokesian viscoelastic flow. Phys. Rev. Lett., 103 (2009), No. 9, 094501. 